\title{
PREVALENCIA Y FACTORES ASOCIADOS AL BAJO CONSUMO DE FRUTAS Y VERDURAS EN ALUMNOS DE LA CARRERA PROFESIONAL DE MEDICINA HUMANA DE UNA UNIVERSIDAD PERUANA
}

PREVALENCE AND FACTORS ASSOCIATED WITH THE LOW CONSUMPTION OF FRUITS AND VEGETABLES IN STUDENTS OF THE PROFESSIONAL CAREER OF HUMAN MEDICINE OF A PERUVIAN UNIVERSITY

Alan Huamancayo-Espíritu', Luis Pérez-Cárdenas'

\begin{abstract}
RESUMEN
Objetivo: Determinar la prevalencia y factores asociados al bajo consumo de frutas y verduras en alumnos de la carrera profesional de medicina humana de la Universidad Nacional Hermilio Valdizán (UNHEVAL). Métodos: Estudio transversal. La población estuvo constituida por los 371 de alumnos de la carrera profesional de Medicina Humana que se encontraban matriculados y cursando estudios en julio de 2018. Se aplicó de un cuestionario autoadministrado el cual permitió obtener información del consumo de frutas y verduras. A partir de los datos recolectados obtuvo la prevalencia y factores asociados al bajo consumo de frutas y verduras. Resultados: La prevalencia de bajo consumo de frutas y verduras fue de $60,1 \%$ (IC95\%: 55,0\%-65,2\%). Las principales razones para no consumir frutas y verduras fueron el considerar que es poco frecuente conseguirlas en los cafetines de la universidad (78,7\%), el tiempo insuficiente para su selección, compra y preparación $(73,6 \%)$ y el vivir con alguno de los padres que se encargaban de su alimentación (55,3\%). El análisis multivariado encontró que el único factor asociado al bajo consumo de frutas y verduras fue el tiempo insuficiente para la selección, compra y preparación; mientras que, vivir con alguno de los padres constituyó factor protector. Conclusión: Existe una alta prevalencia de bajo consumo de frutas y verduras en alumnos de la carrera profesional de medicina humana de la UNHEVAL. El tiempo insuficiente para la selección, compra y preparación de frutas y verduras constituye factor asociado y vivir con alguno de los padres constituye factor protector.
\end{abstract}

Palabras clave: Frutas; Verduras; Estudiantes de medicina (fuente: DeCS BIREME).

\begin{abstract}
Objective: To determine the prevalence and factors associated with the low consumption of fruits and vegetables in students of the professional career of human medicine of the National University Hermilio Valdizán (UNHEVAL). Methods: Cross-sectional study. The population was constituted by 371 students of the professional career of Human Medicine who were enrolled and taking courses in July 2018. It was applied a self-administered questionnaire which allowed to obtain information about the consumption of fruits and vegetables. From the collected data it was obtained the prevalence and factors associated with low consumption of fruits and vegetables. Results: The prevalence of low consumption of fruits and vegetables was $60.1 \%$ (IC95\%: 55.0\% -65.2\%). The main reasons for not consuming fruits and vegetables were that it is rare to get them in the cafeterias of the university $(78.7 \%)$, insufficient time for their selection, purchase and preparation (73.6\%) and living with one of the parents who were in charge for their feeding. (55.3\%). The multivariate analysis found that the only factor associated with low consumption of fruits and vegetables was the insufficient time for selection, purchase and preparation; whereas, living with one of the parents constituted a factor protector. Conclusion: There is a high prevalence of low consumption of fruits and vegetables in students of the professional career of human medicine of the UNHEVAL. The insufficient time for the selection, purchase and preparation of fruits and vegetables is an associated factor and living with one of the parents constitutes a protector factor.
\end{abstract}

Key words: Fruits; Vegetables; Students of Medicine (source: MeSH NLM).

${ }^{1}$ Facultad de Medicina. Universidad Nacional Hermilio Valdizán, Huánuco-Perú.

Citar como: Luis Pérez-Cárdenas, Alan Huamancayo-Espíritu. Prevalencia y factores asociados al bajo consumo de frutas y verduras en alumnos de la carrera profesional de medicina humana de una universidad peruana. Rev. Fac. Med. Hum. Enero 2020; 20(1):123-129. DOI 10.25176/RFMH.v20i1.2660

Artículo publicado por la Revista de la Facultad de Medicina Humana de la Universidad Ricardo Palma. Es un artículo de acceso abierto, distribuído bajo los términos de la Licencia Creative Commons: Creative Commons Attribution 4.0 International, CC BY 4.0 (https://creativecommons.org/licenses/by/4.0/), que permite el uso no comercial, distribución y reproducción en cualquier medio, siempre que la obra original sea debidamente citada. Para uso comercial, por favor póngase en contacto con revista.medicina@urp.pe 


\section{INTRODUCCIÓN}

Las frutas y las verduras constituyen un componente indispensable de una dieta saludable ya que proveen los principales micronutrientes, fibra dietética y otros compuestos esenciales; existe evidencia que el consumo diario de frutas y verduras en cantidades adecuadas se asocia con la reducción del riesgo de desarrollar diversas enfermedades. Por otro lado, un incremento de las frutas y verduras en la dieta contribuye a desplazar alimentos ricos en grasas saturadas, grasas trans, azúcares o sodio. ${ }^{(1-3)}$

Las frutas y verduras constituyen fuente importante de vitaminas, minerales y carbohidratos, aunque las concentraciones de dichos compuestos varían según la fruta o verdura consumida. Además son fuente de fibra, que incluye a la lignina y polisacáridos no almidónicos, así como de principios activos con actividad antioxidante a las que se atribuye un efecto antienvejecimiento y un papel importante en la prevención de enfermedades no transmisibles (ENT). Otro beneficio es la sensación de saciedad, la cual conlleva a una reducción la ingesta de calorías totales y la reducción de la probabilidad de desarrollar sobrepeso y obesidad. ${ }^{(4-7)}$

En la actualidad, el consumo de frutas y verduras varía de región a región (dependiendo de la población

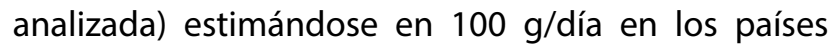
de bajos ingresos y aproximadamente $450 \mathrm{~g} /$ día en Europa Occidental. La ingesta insuficiente de frutas $y$ verduras es responsable de cerca de la quinta parte de las neoplasias gastrointestinales, la tercera parte de las enfermedades isquémicas del corazón y la décima parte de las enfermedades cerebrovasculares. De este modo, cada año podrían salvarse hasta 1 millón 700 mil personas si existiera en el mundo un consumo suficiente de frutas y verduras. ${ }^{(1)}$

Debido a su rol en la prevención de las ENT, la Organización Mundial de la Salud y la Organización de las Naciones Unidas para la Alimentación (FAO) lanzaron en 2003 una iniciativa conocida como "estrategia mundial sobre régimen alimentario, actividad física y salud" con la que esperaban promover el consumo a través del incremento de la producción de frutas y verduras6. El eje central de la iniciativa fue impulsar el consumo de por lo menos cinco porciones diarias de estos alimentos por parte de la población mediante la estrategia conocida como "cinco frutas y verduras al día".(1,8)

La educación universitaria, a la par de brindar competencias para ejercer una profesión, puede generar modificaciones en los estilos de vida de los estudiantes que podrían afectar su alimentación y estado nutricional lo que a su vez tendría repercusiones en el rendimiento académico. Dichas modificaciones pueden ocurrir por la exigencia y competitividad de las carreras universitarias que hacen que los estudiantes dediquen mucho tiempo a ellas descuidando ciertos estilos de vida saludable que de no ser objeto de intervención podrían permanecer más allá de la vida universitaria afectando su riesgo para desarrollar enfermedades en el futuro. ${ }^{(9-12)}$

En el caso de la alimentación de los estudiantes, uno de los aspectos que pueden afectarse es el consumo de frutas y verduras que según algunos estudios podría alcanzar proporciones inferiores a las reportadas en población general. Esto es más relevante en el caso de estudiantes de carreras profesionales de ciencias de la salud quienes durante su práctica profesional deberán promover la alimentación saludable así como otros estilos de vida orientados a la prevención de enfermedades. ${ }^{(6,10,11)}$

La Universidad Nacional Hermilio Valdizán (UNHEVAL) es una universidad peruana que se localiza en la ciudad de Huánuco, la cual se encuentra en la zona central de los Andes a una altitud de 1894 msnm. En 2018, la universidad contaba 14 facultades y 19 carreras profesionales, la carrera profesional de medicina humana contaba con 376 alumnos matriculados.

La presente investigación tiene como objetivo general determinar la prevalencia y factores asociados al bajo consumo de frutas y verduras en alumnos del primer y sexto año de la carrera profesional de medicina humana de la UNHEVAL durante julio de 2018.

\section{MÉTODOS}

Estudio descriptivo de corte transversal. La población estuvo constituida por la totalidad de alumnos del primer al sexto año de estudios de la carrera profesional de Medicina Humana de la UNHEVAL que se encontraban matriculados y cursando estudios en julio de 2018, se excluyó a aquellos que habían reservado matrícula, se habían retirado o que no aceptaron participar voluntariamente del estudio. No se realizó muestreo, se trabajó con la totalidad de la población de alumnos de la carrera profesional de Medicina humana que cumplieron con los criterios de selección por ser esta pequeña y accesible.

La recolección de datos estuvo basada en la aplicación de un cuestionario anautoadministrado a los alumnos el cual permitió obtener información de variables sociodemográficas, del consumo de frutas y verduras y de posibles factores asociados al consumo de estas:

- Variables sociodemográficas: Edad, sexo, año de estudios.

- Consumo de frutas y verduras: Consumo de al menos 5 porciones diarias, número promedio de frutas y verduras consumidas diariamente. 
- Factores asociados al bajo consumo de frutas y verduras: No disponer de dinero suficiente para comprarlas, falta de disponibilidad de frutas y verduras en el campus universitario, tiempo insuficiente para la compra de frutas y verduras, exposición a mensajes en medios de comunicación que recomiendan consumir frutas y verduras.

El cuestionario fue aplicado al término de una clase programada previa coordinación con los delegados del año de estudios y del curso verificándose el correcto llenado de los ítems del previo a su recepción. Se programó una segunda fecha para la aplicación del instrumento orientada a captar a los alumnos que no se encontrasen presentes en la primera aplicación del cuestionario. El cuestionario fue validado mediante apreciación de juicio de expertos obteniéndose el índice de acuerdo y sometido a una prueba piloto para verificar el comportamiento del instrumento en campo así como la comprensibilidad de los ítems por los alumnos.

Con los datos recolectados de cada estudiante se obtuvo la prevalencia de bajo consumo de frutas y verduras en los estudiantes así como los factores asociados al bajo consumo.

El procesamiento de los datos fue realizado con el programa estadístico SPSS versión 23.0 para Windows. Se realizó estadística descriptiva basada en la obtención de frecuencias, porcentajes, medidas de tendencia central (Media, mediana) y de dispersión (Desviación estándar). Para el análisis bivariado se empleó la prueba chi cuadrado de Pearson y para el análisis multivariado se empleó un modelo de regresión logística binaria obteniéndose la razón de posibilidades $u$ odds ratio ajustado (ORAJ). Los cálculos fueron realizados con un nivel de confianza del $95 \%$.

El proyecto fue aprobado por el Comité de Ética de la Dirección de Investigación Universitaria de la UNHEVAL (Resolución Nº 0200-2018-UNHEVAL-FM-D). Se garantizó la confidencialidad de la información obtenida y se respetó los principios éticos de acuerdo con lo estipulado en la Declaración de Helsinki. Previo a la aplicación del cuestionario cada uno de los participantes firmó un consentimiento informado.

\section{RESULTADOS}

De un total de 373 alumnos matriculados que aceptaron participar de la investigación, dos alumnos que cursaban el tercer año de estudios no llenaron el cuestionario completo por lo que quedaron disponibles para el análisis 371 alumnos.

De los 371 alumnos participantes de la investigación, el $52,6 \%$ eran mujeres y el $47,4 \%$ eran varones; asimismo, la mayor frecuencia de alumnos se encontraba cursando el tercer año de estudios (28,6\%). La edad promedio fue de 23,8 \pm 4,3 años (Mediana 24 años) la cual varió entre los 16 y los 46 años de edad. Las características generales de los alumnos se muestran en la tabla 1.

Tabla 1. Características generales de los alumnos de la carrera profesional de Medicina Humana de la UNHEVAL.

\begin{tabular}{lcc}
\hline \multicolumn{1}{c}{ Característica } & Frecuencia & $\%$ \\
\hline Edad & 66 & 17,8 \\
15-19 años & 160 & 43,1 \\
$20-24$ años & 114 & 30,7 \\
$25-29$ años & 27 & 7,3 \\
30-34 años & 2 & 0,5 \\
35-39 años & 1 & 0,3 \\
$40-44$ años & 1 & 0,3 \\
$45-49$ años & & \\
Sexo & 195 & 52,6 \\
Femenino & 178 & 47,4 \\
Masculino & & 16,2 \\
Año de estudios & 60 & 24,8 \\
Primer año & 92 & 28,6 \\
Segundo año & 106 & 12,1 \\
Tercer año & 45 & 8,9 \\
Cuarto año & 33 & 9,4 \\
Quinto año & 35 & \\
Sexto año & &
\end{tabular}


Tabla 2. Prevalencia de bajo consumo de frutas y verduras según año de estudios alumnos de la carrera profesional de Medicina Humana de la UNHEVAL.

Año de estudios

Prevalencia (\%)

46,7

63,0

66,7

62,2

57,6

51,4

60,1

\section{Intervalo de confianza $95 \%$}

$33,2-60,1$

$52,6-73,5$

$57,3-76,0$

$46,9-77,5$

$39,2-76,0$

Sexto año

Primer a sexto año
Las principales razones para no consumir frutas y verduras por parte de los alumnos fueron el considerar que es poco frecuente conseguirlas en los cafetines de la universidad (78,7\%), el tiempo insuficiente para su selección, compra y preparación $(73,6 \%)$ y el vivir con alguno de los padres que se encargan de la alimentación del alumno (55,3\%). Esto se muestra en la tabla 3.

Tabla 3. Razones para el no consumo de frutas y verduras por los alumnos de la carrera de Medicina Humana.

\section{Variable}

Considera que el precio de las frutas y verduras es alto lo que condiciona que no las consuma

Considera que es poco frecuente conseguir frutas y/o verduras en los cafetines de la universidad

Tiempo insuficiente para la selección, compra y preparación de frutas y verduras

Exposición a mensajes en medios de comunicación que recomiendan consumir frutas y verduras

Vivir con alguno de los padres que se encargan de la alimentación del alumno
Frecuencia

160

73,6
Se realizó análisis bivariado con la prueba Chi cuadrado de Pearson encontrándose que el tiempo insuficiente para la selección, compra y preparación de frutas y verduras así como el vivir con alguno de los padres se asociaron significativamente con el bajo consumo de frutas y verduras. La edad menor o igual de 20 años, el sexo, el considerar que el precio de las frutas y verduras es alto, el estudiar en los primeros tres años de la carrera profesional, el considerar que es poco frecuente conseguir frutas y/o verduras en los cafetines de la universidad y la exposición a mensajes en medios de comunicación que recomiendan consumir frutas y verduras no se asociaron significativamente con el bajo consumo de frutas y verduras. Esto se muestra en la tabla 4. 
Tabla 4. Análisis bivariado con la prueba Chi cuadrado de posibles factores de riesgo para bajo consumo de frutas y verduras en estudiantes de la UNHEVAL.

\section{Variable}

Bajo consumo de frutas

$y$ verduras (\%)

Valor $\mathbf{p}$

Considera que el precio de las frutas y verduras es alto lo que condiciona que no las consuma

Sí

$104(65,0 \%)$

0,075

No

$119(55,9 \%)$

Considera que es poco frecuente conseguir frutas y/o verduras en

los cafetines de la universidad

Sí

$176(60,3 \%) \quad 0,715$

No

$47(58,0)$

Tiempo insuficiente para la selección, compra y preparación de

frutas y verduras

Sí

$172(63,0 \%)$

0,036

No

$51(51,0 \%)$

Exposición a mensajes en medios de comunicación que recomiendan consumir frutas y verduras

Sí

$106(58,6 \%)$

0,640

No

$117(60,9 \%)$

Vivir con alguno de los padres que se encargan de la alimentación

del alumno

Sí

$115(55,1 \%)$

0,042

No

$110(65,5 \%)$

Edad menor o igual de 20 años

Sí

$47(53,4 \%)$

0,159

No

$175(61,8 \%)$

Primeros años de estudio

Sí

$158(60,8 \%)$

0,557

No

$65(57,5 \%)$

Las variables que obtuvieron un valor $p \leq 0,10$ fueron ingresadas en un modelo de regresión logística para el análisis multivariado encontrándose que el único factor asociado para el bajo consumo de frutas y verduras fue el tiempo insuficiente para la selección, compra y preparación de frutas y verduras; mientras que, vivir con alguno de los padres que se encargan de la alimentación del alumno constituyó factor protector para el bajo consumo de frutas y verduras (Ver tabla 5).

Tabla 5. Análisis multivariado de posibles factores asociados al bajo consumo de frutas y verduras en alumnos de la carrera profesional de Medicina Humana de la UNHEVAL.

\section{Variable}

Tiempo insuficiente para la selección, compra y preparación de frutas y verduras

Vivir con alguno de sus padres que se encargan de la alimentación del alumno

Considera que el precio de las frutas y verduras es alto lo que condiciona que no las consuma

\section{Valor $\mathbf{p}$}

ORaj

Intervalo de confianza $95 \%$

0,038

1,6

$1,027-2,604$

0,044

0,6

$0,424-0,989$

0,221

1,3

$0,848-2,044$ 


\section{DISCUSIÓN}

La presente investigación muestra que una alta frecuencia de alumnos de la carrera profesional de Medicina Humana de la UNHEVAL presenta bajo consumo de frutas y verduras; asimismo, el único factor asociado al bajo consumo de estas fue el tiempo insuficiente para la selección, compra y preparación de frutas y verduras; mientras que, vivir con alguno de los padres del alumno constituye un factor protector debido a que son ellos los que se encargan de la alimentación del alumno.

Con relación a la prevalencia del bajo consumo de frutas y verduras, se observó que de forma global, seis de cada diez alumnos presentaba dicho factor de riesgo; sin embargo, la prevalencia encontrada es inferior a la reportada por el Instituto Nacional de Estadística e informática(13) en la Encuesta Demográfica y de Salud Familiar (ENDES 2017) para población general peruana de quince a más años donde el bajo consumo de frutas y verduras alcanzó el $89,1 \%$. Esto indica que es posible que los contenidos curriculares de la carrera médica tengan un efecto positivo en el consumo de una fracción de los alumnos, particularmente los contenidos orientados a la nutrición y a la prevención de las ENT. Contrariamente a lo esperado, no se observó una tendencia en el consumo según años de estudio.

Al evaluarse los factores asociados al bajo consumo de frutas y verduras, el análisis multivariado identificó como único factor al tiempo insuficiente para la selección, compra y preparación de frutas y verduras, lo cual ha sido vinculado en otras publicaciones a la carga académica de la carrera, representada por las horas de clase y las horas de estudio(14-17). Esto coincide con lo encontrado por Olivares ${ }^{(15)}$ en un estudio realizado en estudiantes de la Universidad de Chile quienes identifican como la segunda principal barrera para un consumo adecuado de frutas y verduras al no tener tiempo. A diferencia de los alumnos de la Universidad de Chile, la falta de tiempo para la selección, compra y preparación de frutas y verduras en los alumnos de Universidad Nacional Hermilio Valdizán fue notablemente mayor $(63,0 \%$ versus $33,4 \%$ ) lo cual podría explicarse por la alta exigencia de la carrera médica en comparación con las otras carreras profesionales que compusieron la muestra del estudio de Olivares que incluía carreras de salud no médicas, así como carreras de ciencias sociales y ciencias exactas.

El único factor protector identificado por la presente investigación fue el hecho de que el alumno viviera con alguno de sus padres debido a que eran ellos los que se encargaban de su alimentación. Esto se explicaría en lo establecido por algunas publicaciones que consideran que la familia influiría en la forma de alimentarse de los estudiantes, siendo positiva la enseñanza entregada por su núcleo parental, favoreciendo una alimentación saludable al adquirir alimentos con estas características ${ }^{(17-19)}$. Esto coincide con lo reportado por Papadaki y col. ${ }^{(18)}$ quienes encontraron en una muestra de estudiantes universitarios de Grecia que los estudiantes que vivían fuera de casa habían desarrollado hábitos alimenticios más desfavorables que los que vivían en el hogar familiar con alguno de sus padres lo cual indicaba que alejarse del hogar familiar y asumir la responsabilidad de la preparación y compra de alimentos afectaba los hábitos alimenticios de los estudiantes.

El estudio realizado en alumnos de la UNHEVAL ha permitido identificar los factores asociados al bajo consumo de frutas y verduras en los estudiantes lo cual constituye el primer paso para el diseño de una intervención educativa dirigida de forma específica a los estudiantes que permita la mejora de la alimentación por parte de los estudiantes así como la intervención sobre estilos de vida no saludables. Dicha intervención debería contemplar la promoción de la venta de frutas y verduras por los proveedores de alimentos de la universidad lo que tendría efecto positivo en la prevención de ENTy hábitos de los futuros profesionales de la salud. Si bien es cierto que los resultados obtenidos pueden ser extrapolados desde el punto de vista estadístico sólo a los estudiantes de la facultad de medicina humana de la UNHEVAL, es posible que la realidad de los estudiantes sea similar en otras universidades por lo que los resultados obtenidos podrían contribuir con otras universidades para el diseño de futuras intervenciones preventivas.

Una limitación de la presente investigación es que no abordó las diferencias en el consumo de frutas y verduras según el nivel socioeconómico de los estudiantes a diferencia de otras investigaciones 20. Sin embargo, es posible que esto no haya afectado significativamente los resultados obtenidos debido a que se contempló dentro de los factores en estudio la percepción del precio de las frutas y verduras por parte de los estudiantes lo que a su vez condicionaría su consumo.

Otra limitación la constituye el número de estudiantes participantes en el estudio. Así, la estimación de la prevalencia según años de estudio en algunos casos se ve afectada por el bajo número de estudiantes lo cual se refleja en los intervalos de confianza de las estimaciones las cuales se amplían en el caso de los alumnos de quinto y sexto año. Esto no ocurre con la estimación global para los alumnos del primer al sexto 
año donde el número de estudiantes no afecta de forma importante a la estimación de bajo consumo. Debe tomarse en cuenta que la investigación consideró a casi la totalidad de alumnos matriculados por lo que no hubo pérdida importante de estudiantes y que estas son las limitaciones de trabajar con grupos preformados.

\section{CONCLUSIÓN}

La prevalencia de bajo consumo de frutas y verduras en alumnos de la carrera profesional de medicina humana de la Universidad Nacional Hermilio Valdizán fue de $60,1 \%$. El tiempo insuficiente para la selección, compra y preparación de frutas y verduras constituye factor asociado al bajo consumo; mientras que, vivir con alguno de los padres constituye factor protector.
Contribuciones de autoría: Los autores participaron en la génesis de la idea, diseño de proyecto, recolección e interpretación de datos, análisis de resultados, preparación del manuscrito.

Financiamiento: Autofinanciado.

Conflicto de interés: Los autores declaran no tener ningún conflicto de interés.

Recibido: 10 de octubre del 2019

Aprobado: 18 de diciembre del 2019

\section{Correspondencia: Alan Huamancayo Espíritu.}

Dirección: Av. Vía colectora Mz A Lt 4. Pillcomarca, Huánuco-Perú.

Teléfono: +51962629911

Correo:alan.smill7@gmail.com

\section{REFERENCIAS BIBLIOGRÁFICAS}

1. Organización Mundial de la Salud. Estrategia mundial sobre régimen alimentario, actividad física y salud. Ginebra: OMS; 2016. Obtenido de: http:// www.who.int/dietphysicalactivity/fruit/es/index1.html.

2. Miller V, Yusuf S, Chow C. Availability, affordability, and consumption of fruits and vegetables in 18 countries across income levels: findings from the Prospective Urban Rural Epidemiology (PURE) study. Lancet Glob Health, 2016;4(10):e695-e703.

3. Jacoby E, Keller I. La promoción del consumo de frutas y verduras en américa latina: buena oportunidad de acción intersectorial por una alimentación saludable. Rev chil Nutr 2006;33(Supl. 1):226-31.

4. Centers for Disease Control and Prevention. Can eating fruits and vegetables help people to manage their weight? Atlanta: Research to Practice Series N 1; 2005. Disponible en: http://www.cdc.gov/nccdphp/dnpa/nutrition/pdf/ rtp_practitioner_10_07.pdf.

5. Aune D, Greenwod DC. Dietary fibre, whole grains, and risk of colorectal cancer: systematic review and dose-response meta-analysis of prospective studies. BMJ 2011;343:d6617.

6. Acosta B. Promoción del consumo de frutas y verduras en grupos representativos de la Parroquia Nanegal, Provincia, Pichincha, Periodo Noviembre Diciembre del 2014. [Internet]. Pontifica de Universidad Católica del Ecuador; 2014

7. Correa A. Consumo de frutas y verduras y hortalizas en Adolescentes durante el ciclo secundario de enseñanza. Universidad Abierta Interamericana; 2005.

8. Byers T, Nestle M, McTiernan A, Doyle, C, Currie-Williams A, Gansler T, et al. American Cancer Society guidelines on nutritionmand physical activity for cancer prevention: reducing the risk of cancer with healthy choices and physical activity. CA Cancer. J Clin. 2002;52:92-119.

9. Vargas M, Becerra F, Prieta E. Evaluación de la ingesta dietética en estudiantes universitarios. Bogotá, Colombia. Rev Salud Pública. 2010;12:116-25.

10. Becerra-Bulla $F$, Vargas-Zárate $M$. Estado nutricional y consumo de alimentos de estudiantes universitarios admitidos a nutrición y dietética en la Universidad Nacional de Colombia. Rev. salud pública. 2015;17(5):762-75.
11. Kongsbak IS, Nielsen BK, Ahlmann FK, Schaldemose $H$, Atkinson L, Wichmann $M$, et al. Increasing fruit and vegetable intake among male university students in an ad libitum buffet setting: A choice architectural nudge intervention. Food Quality and Preference, 2016;9; 183-8.

12. Zazpe I, Marqués M, Sánchez-Tainta A, Rodríguez-Mourille A, Beunza JJ, Santiago $S$, et al. Hábitos alimentarios y actitudes hacia el cambio en alumnos y trabajadores universitarios españoles. Nutr Hosp. 2013;28(5):1673-1680

13. Instituto Nacional de Estadística e Informática (INEI). Perú, Enfermedades no transmisibles y transmisibles, 2017. Lima: INEI, 2018. Disponible en https://www.inei.gob.pe/media/MenuRecursivo/publicaciones digitales/Est/ Lib1526/index.html.

14. Rosales Ramírez MF. Efectos de la publicidad presente en los medios de comunicación, en la alimentación de jóvenes universitarios. 2017. Tesis Doctoral. Concepción: Universidad Católica de la Santísima Concepción; 2017.

15. Olivares S, Lera L, Bustos N. Etapas del cambio, beneficios y barreras en actividad física y consumo de frutas y verduras en estudiantes universitarios de Santiago de Chile. Rev. chil. nutr. 2008;35(1):25-35.

16. Lema Soto LF, Salazar Torres IC, Varela Arévalo MT, Tamayo Cardona JA Rubio Sarria A, Botero Polanco A. Comportamiento y salud de los jóvenes universitarios: satisfacción con el estilo de vida. Pensamiento Psicológico 2009;5(12):71-88.

17. Troncoso C, Amaya JP. Factores sociales en las conductas alimentarias de estudiantes universitarios. Rev. chil. nutr. 2009;36(4):1090-7.

18. Papadaki A, Hondros GA Scott J, Kapsokefalou M. Eating habits of university students living at, or away from home in Greece. Appetite 2007;49(1)169-76.

19. Ponce PG, Ruiz Esparza CJ, Magaña RM, Arizona AB, Mayagoitia WJ. Obesidad y factores de riesgo en estudiantes del área de la salud de la Universidad Autónoma de Baja California, Mexicali. Rev Salud Pública Nutr. 2011;12(4).

20. Restrepo LF, Urango LA, Deossa GC. Conocimiento y factores asociados al consumo de frutas por estudiantes universitarios de la ciudad de Medellín, Colombia. Rev Chil Nutr 2014:41(3):236-42. 